\title{
The Special Features of the Iraqi Distribution Electric Power Networks
}

\author{
Michael A. Averbukha ${ }^{a}$ Mohammed W. Abdulwahhaba,c, \\ Eugene V. Zhilin ${ }^{\mathrm{a}}$ and Evgenia Yu. Sizganova ${ }^{\mathrm{b} *}$ \\ ${ }^{a}$ Belgorod State Technological University named after V.G. Shukhov \\ 46 Kostyukova Str., Belgorod, 308012, Russia \\ ${ }^{b}$ Siberian Federal University \\ 79 Svobodny, Krasnoyarsk, 660041, Russia \\ ${ }^{c}$ Daiyla University \\ Baquba City, Iraq
}

Received 13.05.2019, received in revised form 29.05.2019, accepted 10.06.2019

The paper examines the impact of distributed generation facilities on the operating parameters of Iraq's 33/11 kV distribution networks. The performed calculation of steady-state and post-emergency modes in the RastrWin3 software package showed that the networks are overloaded and therefore the voltage and power losses in the nodes and branches reach their limits. It was revealed that the most optimal technical measure that increases the voltage level in the load nodes and reduces power losses in the branches is the use of distributed generation, which includes diesel power plants and solar plants. The calculated operation modes considering connection of photo-diesel power stations to the network showed that the voltage and power losses in the nodes and branches of the circuit were significantly reduced.

Keywords: distribution networks, distributed generation, photo-diesel power stations, power and voltage losses.

Citation: Averbukh M.A., Abdulwahhab M.W., Zhilin E.V., Sizganova E.Yu. The special features of the iraqi distribution electric power networks, J. Sib. Fed. Univ. Eng. technol., 2019, 12(5), 607-616. DOI: 10.17516/1999-494X-0160.

(C) Siberian Federal University. All rights reserved

This work is licensed under a Creative Commons Attribution-NonCommercial 4.0 International License (CC BY-NC 4.0).

* Corresponding author E-mail address: seu_eset@mail.ru 


\title{
Особенности режимов распределительных
}

\section{электроэнергетических сетей Ирака}

\author{
М.А. Авербух ${ }^{\mathrm{a}}$, М.В. Абдулваххаб ${ }^{\mathrm{a}, \mathrm{s}}$, \\ Е.В. Жилин ${ }^{a}$, Е.Ю. Сизганова ${ }^{6}$ \\ ${ }^{a}$ Белгородский государственный технологический университет \\ им. В.Г. Шухова \\ Россия, 308012, Белгород, ул. Костюкова 46 \\ ${ }^{6}$ Сибирский федеральный университет \\ Россия, 660041, Красноярск, пр. Свободныий, 79 \\ ${ }^{6}$ Университет Дияльь \\ Ирак, Дияла
}

В работе рассматривается влияние распределенной генерации на параметры режима работы распределительных сетей Ирака 33/11 кВ. Выполнен расчет установившегося и послеаварийного режимов работы в программном комплексе RastrWin3, который показал, что сети перегружены, и в связи с этим потери напряжения и мощности в узлах и ветвях достигают предельных значений. Выявлено, что наиболее оптимальным техническим мероприятием, повышающим уровень напряжения в узлах нагрузки и снижающим потери мощности в ветвях, является использование распределенной генерации, включающей в себя дизельные электростаниии и фотоэлементы. Произведен расчет режимов работы с использованием фотодизельных электростанций, которыепоказали, что потеринапряжения и мощности в узлах и ветвях схемь значительно снизились.

Ключевые слова: распределительные сети, распределенная генерачия, фотодизельные электростанщии, потери мощности и напряжения.

В результате военных действий энергетическая система и распределительные сети Республики Ирак существенно пострадали, а оставшиеся в работе сети значительно перегружены. Так, например, спрос электроэнергии в Ираке на 2018 г. составлял 18000 МВт, в то время как энергосистема вырабатывает 15500 МВт. Перегрузка распределительных сетей приводит к множеству негативных явлений, таких так отклонение напряжения в узлах нагрузок, увеличение потерь мощности и электроэнергии; снижение срока службы электрооборудования распределительной сети [1-6].

Фрагмент распределительной сети напряжением 33 кВ с подстанциями 33/11 кВ представлен на рис. 1.

Распределительные сети Ирака включают в себя более 200 подстанций напряжением 132/33 кВ общей мощностью 27000 МВА и сети напряжением 33 кВ с большим количеством подстанций напряжением 33/11 кВ. Протяженность сетей напряжением 33 кВ составляет порядка 12000 км, а суммарная протяженность линий напряжением 11 кВ - 52000 км [7, 8].

Как следует из данных рис. 1, теплоэлектростанции являются источником электроэнергии для распределительных сетей 33 кВ, к которым подключены двухтрансформаторные подстанции напряжением 33/11 кВ. Они образуют узлы нагрузок, питающие города и промышленные объекты. Представленный фрагмент характерен для всей системы электроснабжения Ирака.

Для расчетов нормального режима работы распределительных сетей на основании рис. 1 составлена схема замещения. 


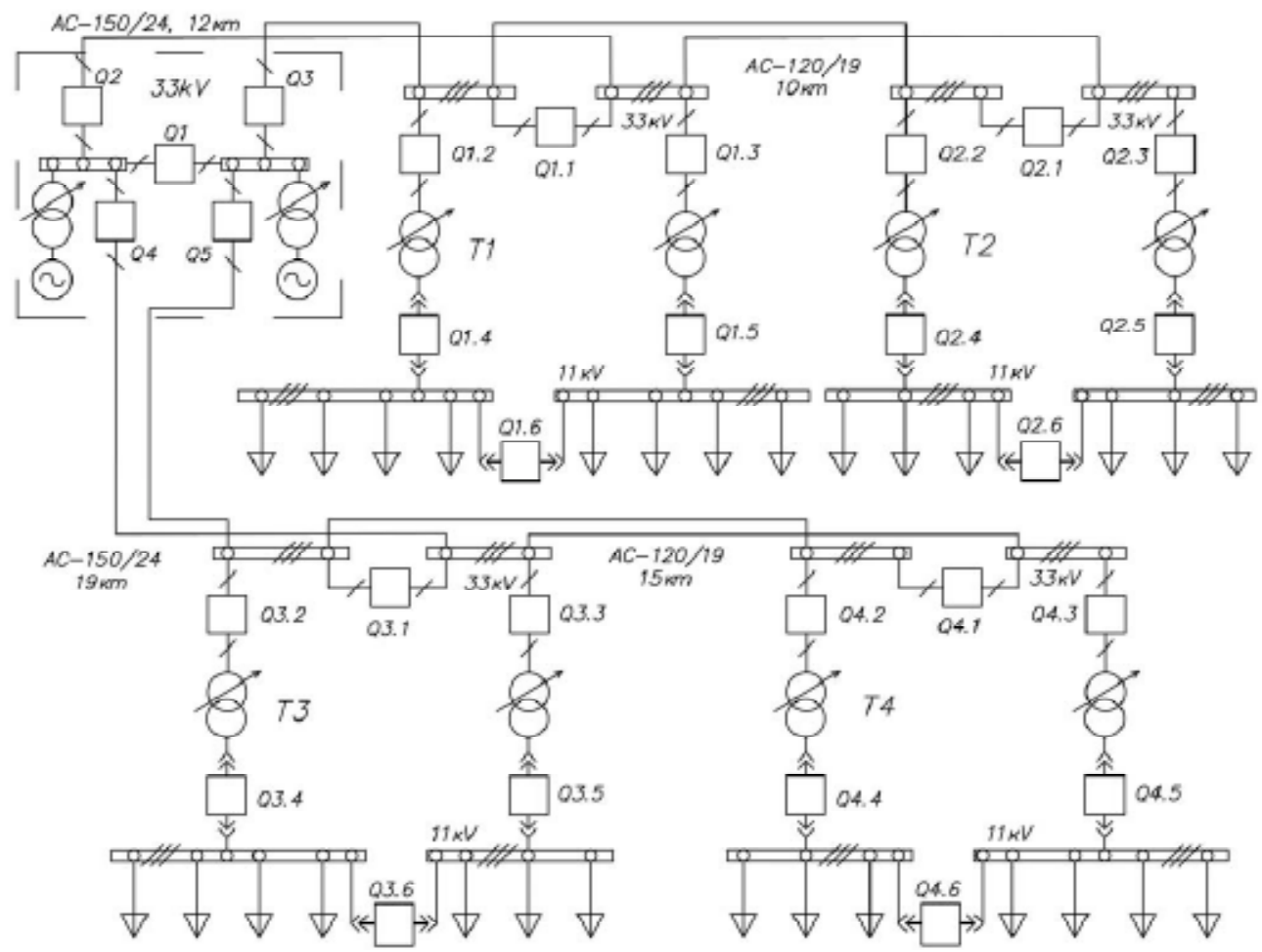

Рис 1. Фрагмент распределительной сети напряжением 33/11 кВ системы электроснабжения Ирака

Fig. 1. Fragment of a 33/11 kV distribution network in Iraq

Расчет элементов схемы замещения произведен на основании следующих выражений $[9,10]$ : воздушная линия электропередач (ЛЭП):

$$
R=R_{0} \cdot L / n ; X=X_{0} \cdot L / n ; B=n \cdot B_{0} \cdot L,
$$

где $\mathrm{L}$ - длина линии, км; $\mathrm{R}_{0}$ и $\mathrm{X}_{0}$ - удельные активное и реактивное сопротивления ЛЭП, соответственно, Ом/км; $\mathrm{B}_{0}$ - удельная проводимость ЛЭП, См/км; n - число цепей ЛЭП; трансформатор:

$$
R_{\mathrm{T}}=\frac{\Delta P_{\mathrm{K}} \cdot U_{\mathrm{BH}}^{2}}{n \cdot S_{\mathrm{H}}^{2}} ; X_{\mathrm{T}}=\frac{u_{\mathrm{K}} \cdot U_{\mathrm{BH}}^{2}}{100 \cdot n \cdot S_{\mathrm{H}}} ; G_{T}=\frac{n \cdot \Delta P_{X}}{U_{\mathrm{BH}}^{2}} ; B_{X}=\frac{n \cdot \Delta Q_{X}}{U_{\mathrm{BH}}^{2}},
$$

где $\Delta \mathrm{P}_{\mathrm{K}}$ - активные потери мощности короткого замыкания, кВт; $\Delta \mathrm{P}_{\mathrm{X}}$ - активные потери мощности холостого хода, кВт; $\Delta \mathrm{Q}_{\mathrm{x}}$ - реактивные потери мощности холостого хода, квар; $\mathrm{U}_{\mathrm{BH}}-\mathrm{Ho}-$ минальное напряжение первичной обмотки, кB; $\mathrm{S}_{\mathrm{H}}-$ номинальная мощность, кBA; $\mathrm{u}_{\mathrm{\kappa}}-$ напряжение короткого замыкания, \%; $\mathrm{n}$ - число параллельно работающих трансформаторов.

На рис. 2 представлена однолинейная схема замещения распределительной сети Ирака 33/11 кВ.

Расчет режимов распределительной сети произведен с помощью программного комплекса RastrWin3, который позволяет проводить не только расчет, но и анализ режимов работы сетей различных уровней напряжения и конфигураций. Пользовательский интерфейс RastrWin3

$$
-609-
$$




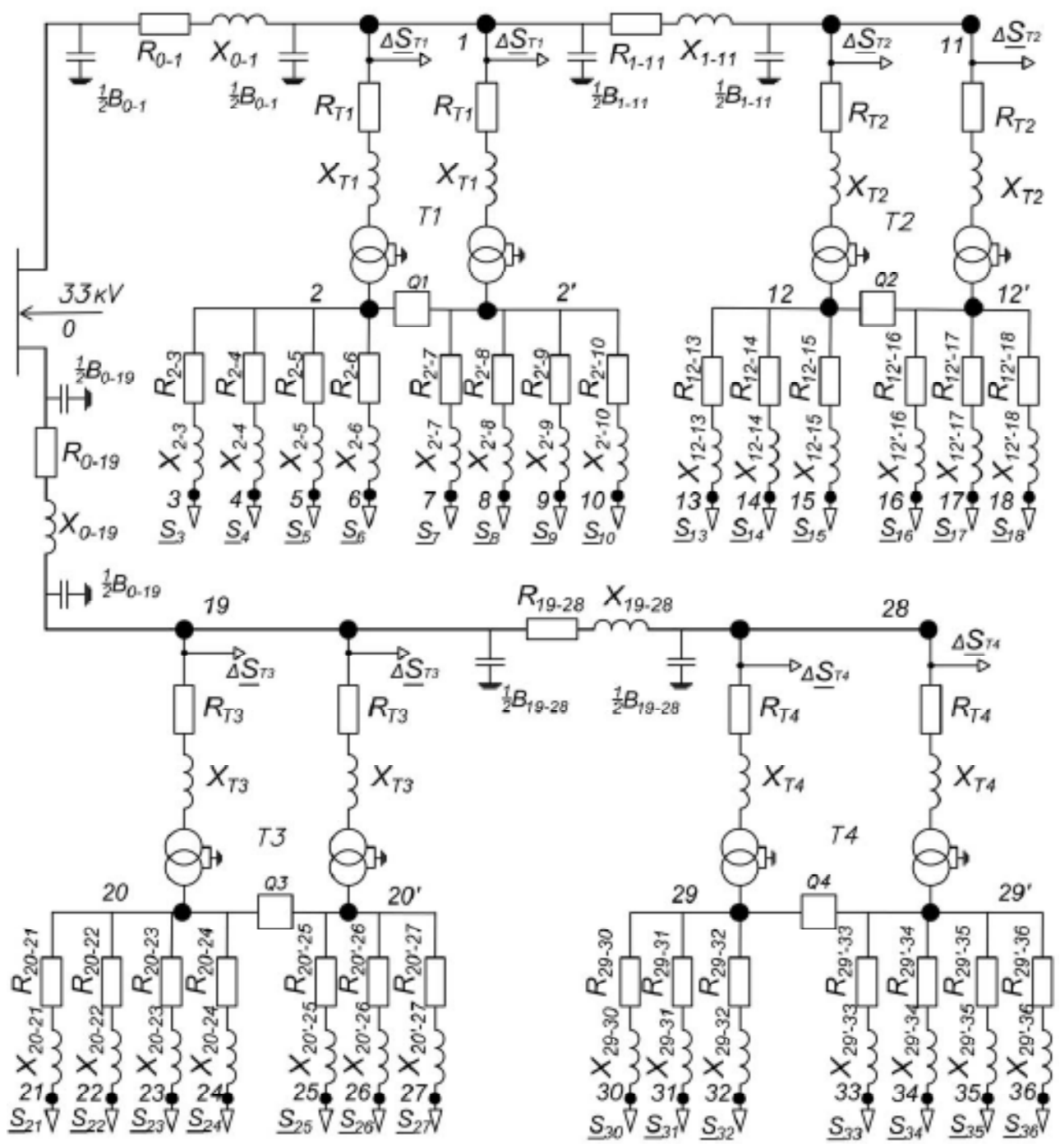

Рис. 2. Однолинейная схема замещения распределительной сети напряжением 33/11 кВ

Fig. 2. The single line equivalent circuit of a $33 / 11 \mathrm{kV}$ distribution network

представляет из себя табличный процессор, поддерживающий возможность ввода, группового или одиночного редактирования данных, сортировку, а также графические средства анализа результатов расчета [11].

Исходными данными для расчетов режимов в программном комплексе RastrWin3 являются: топология схемы (направленный граф); параметры ветвей и трансформаторов схемы замещения; мощности в узлах нагрузок; номинальное напряжение источника энергии; коэффициенты трансформации. За базисный узел принимается нулевой узел на шинах генераторного напряжения.

Результаты расчета нормального и послеаварийного режима работы представлены в табл. 1. Аварийным режим работы - отключение одного из трансформаторов и включение секционного выключателя на шинах 11 кВ. 
Как следует из данных табл. 1, в узлах 3-10 происходит значительное снижение напряжения, до $40 \%$ от номинального, что подтверждает перегруженность распределительных сетей. Потери мощности в ветвях электроэнергетической системы достигают $20 \%$ от потребляемой электроэнергии. Это также связано со значительной перегрузкой электроэнергетической системы. Расчеты показали, что необходимо принятие дополнительных технических решений по снижению потерь мощности в распределительных сетях путем повышения пропускной способности или использования распределенной генерации.

На рис. 3 представлена классификация технических мероприятий, повышающих уровень напряжения в узлах нагрузок и снижающих потери мощности [12-18].

Таблица 1. Результаты расчета нормального и послеаварийного режимов в распределительных сетях

Table 1. A results of the normal calculation and post-accident conditions in distribution networks

\begin{tabular}{|c|c|c|c|c|c|c|c|c|}
\hline \multicolumn{9}{|c|}{ Параметры узлов } \\
\hline \multirow{2}{*}{ № узла } & \multicolumn{3}{|c|}{ Нормальный } & \multicolumn{2}{|c|}{ Послеаварийный } & \multirow{2}{*}{\multicolumn{2}{|c|}{$\mathrm{P}_{\mathrm{p}}, \mathrm{MB} \mathrm{T}$}} & \multirow{2}{*}{$\mathrm{Q}_{\mathrm{p}}$, Мвар } \\
\hline & \multicolumn{2}{|c|}{$\mathrm{U}$, кВ } & $\delta \mathrm{U}, \%$ & $\mathrm{U}$, кB & $\delta \mathrm{U}, \%$ & & & \\
\hline 2 & \multicolumn{2}{|c|}{10,20} & 7,30 & 8,34 & \multicolumn{2}{|l|}{24,16} & - & - \\
\hline 2 ' & \multicolumn{2}{|c|}{10,28} & 6,35 & - & \multicolumn{2}{|l|}{-} & - & - \\
\hline 3 & \multicolumn{2}{|c|}{9,52} & 13,41 & 7,49 & \multicolumn{2}{|l|}{31,93} & 1,17 & 0,53 \\
\hline 4 & \multicolumn{2}{|c|}{9,31} & 15,39 & 7,19 & \multicolumn{2}{|l|}{31,65} & 1,43 & 0,59 \\
\hline 5 & \multicolumn{2}{|c|}{8,77} & 20,32 & 6,38 & 42,04 & & & 0,89 \\
\hline 6 & 9,3 & & 14,94 & 7,26 & 34,00 & & & 0,93 \\
\hline 7 & 9,2 & & 16,23 & 6,91 & 37,14 & & & 0,62 \\
\hline 8 & 9,2 & & 15,55 & 7,03 & 36,04 & & & 0,72 \\
\hline 9 & 9,3 & & 14,79 & 7,15 & 35,00 & & & 0,76 \\
\hline 10 & 9,3 & & 14,63 & 7,18 & 34,76 & & & 0,86 \\
\hline & & & & яетры в & & & & \\
\hline & & $\mathrm{Ho}$ & альный & & & Посл & ийный & \\
\hline № ветви & $\begin{array}{c}\mathrm{S}_{\text {iјначала }} \\
\mathrm{MBA}\end{array}$ & $\mathrm{I}, \mathrm{A}$ & $\Delta \mathrm{P}$, кВТ & $\delta \mathrm{U}, \%$ & $\begin{array}{c}\mathrm{S}_{\text {ijначала }} \\
\mathrm{MBA}\end{array}$ & $\mathrm{I}, \mathrm{A}$ & $\Delta \mathrm{P}, \kappa \mathrm{BT}$ & $\delta \mathrm{U}, \%$ \\
\hline $0-1$ & $21,4+14,3$ & 426 & 832 & 6,27 & $23,2+21,4$ & 553 & 1401 & 9,19 \\
\hline $1-2$ & $6,7+4,6$ & 142 & 57 & 4,74 & $14,6+12,8$ & 373 & 400 & 14,07 \\
\hline $1-2^{\prime}$ & $7+4,4$ & 147 & 61 & 4,85 & - & - & - & - \\
\hline $2-3$ & $1,2+0,6$ & 78 & 60 & 6,11 & $1,3+0,7$ & 99 & 96 & 7,77 \\
\hline $2-4$ & $1,5+0,7$ & 96 & 99 & 8,08 & $1,6+0,8$ & 123 & 163 & 10,49 \\
\hline $2-5$ & $1,9+1,2$ & 125 & 199 & 13,01 & $2+1,4$ & 170 & 366 & 17,88 \\
\hline $2-6$ & $2+1,1$ & 132 & 125 & 7,63 & $2,1+1,2$ & 168 & 205 & 9,84 \\
\hline $2^{\prime}-7$ & $1,7+0,8$ & 107 & 134 & 9,69 & $1,8+0,9$ & 141 & 234 & 12,98 \\
\hline $2^{\prime}-8$ & $1,4+0,9$ & 93 & 103 & 9,01 & $1,5+1$ & 122 & 176 & 11,90 \\
\hline $2^{\prime}-9$ & $1,8+0,9$ & 116 & 121 & 8,25 & $1,9+1$ & 151 & 205 & 10,84 \\
\hline $2^{\prime}-10$ & $2+1$ & 125 & 127 & 8,09 & $2+1,2$ & 162 & 214 & 10,60 \\
\hline
\end{tabular}

Примечание: 2' - номер узла, который в аварийном режиме при срабатывании секционного выключателя совмещается с узлом 2 . 


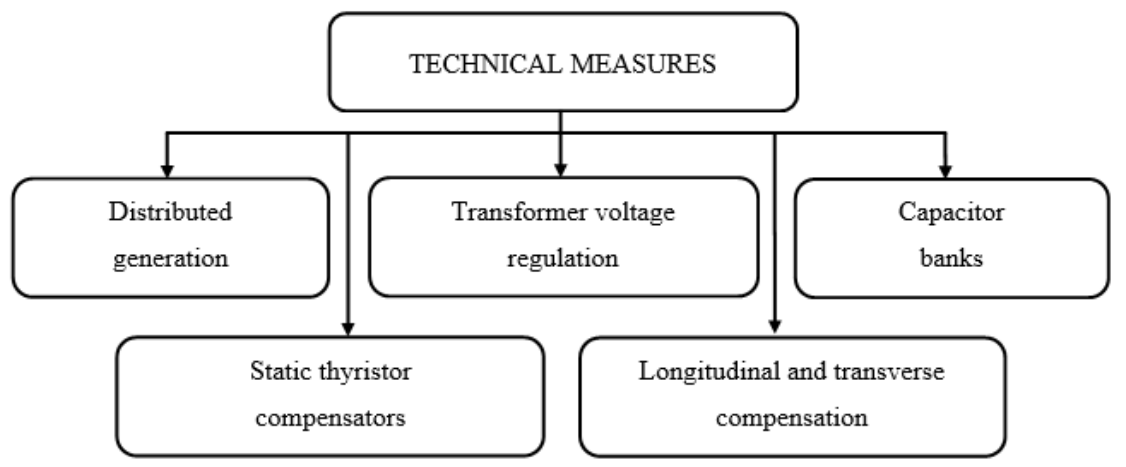

Рис. 3. Технические мероприятия, повышающие уровень напряжения в узлах нагрузки и снижение потерь мощности в распределительных сетях

Fig. 3. Technical measures that increase the voltage in the load nodes and reduce power losses in the distribution networks

Применение батарей статических конденсаторов позволяет снизить перетоки реактивной мощности в сети, что приводит к уменьшению потерь активной энергии в сетях 11-33 кВ, а это в свою очередь дает возможность снизить загрузку ЛЭП и трансформаторов. Продольная компенсация используется для уменьшения реактивного сопротивления линии. Компенсация проводится последовательным включением в рассечку ЛЭП батареи конденсаторов. При такой компенсации батареи конденсаторов подключаются на шины 11 кВ параллельно нагрузке, что приводит к уменьшению потерь мощности и электроэнергии. Но уменьшение передаваемой реактивной мощности снижает потери энергии незначительно, так как активная передаваемая мощность не уменьшается.

Регулирование напряжения трансформатора при использовании устройства регулирования под нагрузкой (РПН) применяется для повышения напряжения с высокой стороны трансформатора, что увеличивает напряжение в узлах нагрузки, но не снижает значение передаваемых мощностей. Поэтому использование РПН снижает уровень потерь напряжения, но не приводит к разгрузке ЛЭП.

Статический тиристорный компенсатор представляет собой устройство для плавного регулирования реактивной мощности и удержания ее значения в заданных пределах. Его принцип работы основан на параллельном включении в сеть фильтрокомпенсирующих цепей, выполняющих функцию фильтров высших гармоник. Данное техническое решение оправданно при значительном преобладании нелинейных потребителей, но не влияет на значение передаваемой мощности.

С учетом климатических особенностей Ирака (протяженность распределительных сетей, удаленные труднодоступные районы с неразвитой инфраструктурой, высокая стоимость строительства дополнительных ЛЭП) наиболее целесообразным техническим решением по снижению загруженности распределительных сетей $33 / 11$ кВ является применение распределенной генерации. Дополнительными факторами целесообразности использования распределенной генерации служит невысокая стоимость дизельного топлива и достаточно высокий уровень солнечного излучения. Зарубежный и российский опыт свидетельствует, что в качестве распределенной генерации нашли широкое применение фотодизельные электроэнергетические 
системы $[19,20]$. Пример использования таких систем показал, что они способны решать следующие задачи: снижать капитальные затраты на строительство новых ЛЭП и уменьшать финансовые риски, вводить дополнительные мощности взамен выбывающих, снижать затраты на потери мощности и электроэнергии в элементах распределительных сетей [21, 22].

Существуют различные схемы подключения распределенной генерации. На рис. 4. представлены схемы подключения фотоэлектрических панелей (ФП) и дизельной электростанции (ДЭС) к системе электроснабжения [23, 24]: раздельная работа ДЭС и ФП с накопителем электроэнергии (НЭ), подключенных через батарейный инвертор (рис. 4а); подключение через сетевой инвертор ФП параллельно с локальной электрической сетью, образованной ДЭС (рис. 4б); раздельная и совместная работа ФП и ДЭС с использованием НЭ и гибридного инвертора (рис. 4в).

В качестве примера использования распределенной генерации рассмотрен узел 2. Дефицит активной мощности для данного узла составил 2000 кВт. Принимаем к установке дизельэлектростанцию Marelli MJB 400-LB4 мощностью 1500 кВт и фото панели Yingli Solar Panda YL275C-30b суммарной мощностью 500 кBт [25]. В программе RastrWin3 произведен расчет режимов на основании схемы замещения (рис. 2) с учетом установленной распределенной генерации.

Результаты расчета нормального и послеаварийного режима работы с использованием распределенной генерации представлены в табл. 2.

Сравнительный анализ результатов расчета, представленных в табл. 1 и 2, показал, что применение распределенной генерации в узле 2 снизило потери напряжения в узлах нагрузки, следующих за вторым узлом, более чем на $10 \%$. При этом ток и потери мощности в распределительной сети напряжением 33 кВ уменьшились примерно на $40 \%$.

\section{Выводы}

1. Расчет нормального и послеаварийного режимов работы распределительных сетей Ирака напряжением 33/11 кВ показал, что сети перегружены и в связи с этим потери на-

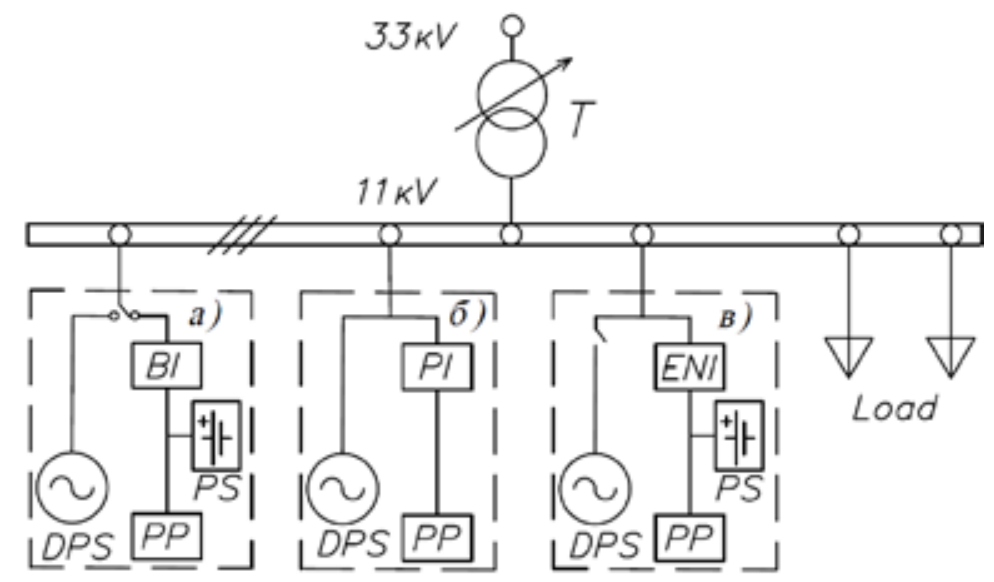

Рис. 4. Схемы подключения фотодизельных электростанций

Fig. 4. Connection schemes of the photo-diesel powerhouses; PP -photovoltaic panels; DPS - diesel power station; PS - power storage; BI - battery inverter; ENI - electric network inverter 
Таблица 2 Результаты расчета нормального и послеаварийного режимов в распределительных сетях с использованием распределенной генерации

Table 2. A results of the normal calculation and post-accident modes in the distribution networks with using distributed generation

\begin{tabular}{|c|c|c|c|c|c|c|c|c|}
\hline \multicolumn{9}{|c|}{ Параметры узлов с использованием распределенной генерации } \\
\hline \multirow{2}{*}{ № узла } & \multicolumn{3}{|c|}{ Нормальный } & \multicolumn{2}{|c|}{ Послеаварийный } & \multirow{2}{*}{\multicolumn{2}{|c|}{$\mathrm{P}_{\mathrm{p}}, \mathrm{MBT}$}} & \multirow{2}{*}{$\mathrm{Q}_{\mathrm{p}}$, Мвар } \\
\hline & \multicolumn{2}{|c|}{$\mathrm{U}$, кВ } & $\delta \mathrm{U}, \%$ & $\mathrm{U}, \kappa \mathrm{B}$ & $\delta \mathrm{U}, \%$ & & & \\
\hline 2 & \multicolumn{2}{|c|}{11,16} & 1,46 & 9,58 & \multicolumn{2}{|c|}{12,87} & - & - \\
\hline 2 & \multicolumn{2}{|c|}{11,26} & 2,35 & - & \multicolumn{2}{|l|}{-} & - & - \\
\hline 3 & \multicolumn{2}{|c|}{10,56} & 4,01 & 8,86 & \multicolumn{2}{|l|}{19,43} & 1,17 & 0,53 \\
\hline 4 & \multicolumn{2}{|c|}{10,37} & 5,74 & 8,62 & \multicolumn{2}{|l|}{21,59} & 1,43 & 0,59 \\
\hline 5 & \multicolumn{2}{|c|}{9,90} & 9,99 & 8,02 & \multicolumn{2}{|l|}{27,09} & 1,68 & 0,89 \\
\hline 6 & \multicolumn{2}{|c|}{10,41} & 5,36 & 8,68 & \multicolumn{2}{|l|}{21,10} & 1,92 & 0,93 \\
\hline 7 & \multicolumn{2}{|c|}{10,31} & 6,28 & 8,42 & \multicolumn{2}{|l|}{23,50} & 1,59 & 0,62 \\
\hline 8 & 10 , & & 5,71 & 8,50 & 22,72 & & & 0,72 \\
\hline 9 & 10 , & & 5,03 & 8,59 & 21,87 & & & 0,76 \\
\hline 10 & 10 , & & 4,90 & 8,61 & 21,69 & & & 0,86 \\
\hline & Па & гетры & твей с испо & ование & спределен & й гене & & \\
\hline & & $\mathrm{Ho}$ & альный & & & Посл & ийный & \\
\hline № ветви & $\begin{array}{c}\mathrm{S}_{\text {iјначала }} \\
\mathrm{MBA}\end{array}$ & $\mathrm{I}, \mathrm{A}$ & $\Delta \mathrm{P}$, кВт & $\delta \mathrm{U}, \%$ & $\begin{array}{c}\mathrm{S}_{\text {iјначала }} \\
\mathrm{MBA}\end{array}$ & $\mathrm{I}, \mathrm{A}$ & $\Delta \mathrm{P}$, кВт & $\delta \mathrm{U}, \%$ \\
\hline $0-1$ & $12,3-0,3$ & 205 & 192 & 1,46 & $17,2+8,5$ & 336 & 517 & 4,74 \\
\hline $1-2$ & $4,6+0,7$ & 79 & 18 & 0,94 & $11,9+6,6$ & 250 & 0,179 & 7,18 \\
\hline $1-2^{\prime}$ & $4,9+0,8$ & 83 & 20 & 1,01 & - & - & - & - \\
\hline $2-3$ & $1,2+0,6$ & 70 & 49 & 5,50 & $1,2+0,6$ & 84 & 70 & 6,56 \\
\hline $2-4$ & $1,5+0,7$ & 86 & 80 & 7,24 & $1,5+0,8$ & 103 & 115 & 8,72 \\
\hline $2-5$ & $1,8+1,1$ & 111 & 156 & 11,48 & $1,9+1,2$ & 137 & 236 & 14,22 \\
\hline $2-6$ & $2+1,1$ & 118 & 101 & 6,85 & $2,1+1,1$ & 142 & 145 & 8,23 \\
\hline 2'-7 & $1,7+0,8$ & 96 & 107 & 8,63 & $1,7+0,8$ & 117 & 160 & 10,63 \\
\hline 2'-8 & $1,4+0,8$ & 84 & 82 & 8,06 & $1,4+0,9$ & 102 & 122 & 9,85 \\
\hline 2'-9 & $1,8+0,9$ & 104 & 98 & 7,39 & $1,9+1$ & 126 & 144 & 9,00 \\
\hline 2'-10 & $1,9+1$ & 112 & 102 & 7,25 & $2+1,1$ & 136 & 150 & 8,82 \\
\hline
\end{tabular}

пряжения и мощности в узлах и ветвях достигают предельных величин. Так, например, потери напряжения между узлами 2-5 составляют $\delta U=20,3$ \%, а потери мощности в ветви $-\Delta \mathrm{P}=199$ кВт, что свидетельствует о перегрузке распределительных сетей и необходимости принятия соответствующих технических решений по изысканию резервных мощностей.

2. Анализ технических мероприятий, повышающих уровень напряжения в узлах нагрузки и снижающих потери мощности в распределительных сетях, показал, что с учетом климатических особенностей Ирака и стоимости дизельного топлива наиболее рациональным вариантом снижения потерь мощности и напряжения является использование распределенной генерации, включающей в себя дизельные электростанции и фотоэлементы. 


\section{Список литературы}

[1] Фурсанов М.И., Золотой А.А., Макаревич В.В. Учет потребительских энергоисточников в расчетах распределительных электрических сетей 6-10 кВ. Энергетика. Известия высших учебных заведений и энергетических объединений СНГ, 2011, 4, 11-15 [Fursanov M.I., Golden A.A., Makarevich V.V. Accounting for consumer energy sources in the calculations of distribution networks 6-10 kV. Energy. Proceedings of higher educational institutions and energy associations of the CIS, 2011, 4, 11-15 (in Russian)].

[2] Авербух М.А., Жилин Е.В. Влияние нелинейной и несимметричной нагрузки на систему электроснабжения жилых микрорайонов. Промышленная энергетика, 2017, 12, 40-45 [Averbukh M.A., Zhilin E.V. Influence of nonlinear and asymmetric load on the power supply system of residential areas. Industrial energy, 2017, 12, 40-45 (in Russian)]

[3] Wang X.F., Song Y., Irving M. Modern Power Systems Analysis. Springer-Verlag New York, Inc., 2008. $561 \mathrm{p}$.

[4] Averbukh M.A., Zhilin E.V., Roschubkin P.V. Experimental analysis of electrical modes in a residential estate electrical power supply system. J. of Engineering and Appl. Sciences, 2017, 12, 34463451.

[5] Ramachandra Murthy K.V.S., Ramalinga Raju M. Electrical energy loss in rural distribution feeders, a case study. ARPN Journal of Engineering and Applied Sciences, 2009, 4(2), 33-37.

[6] Costa P.M., Matos M.A. Loss allocation in distribution networks with embedded generation. IEEE transactions on power systems, 2004, 19(1) 384-389.

[7] Rashid S. Electricity Problem in Iraq. Hamburg, 2012, 22 p.

[8] Larkin L. Iraq's electricity master plans. Iraq future energy, 2011, 69 p.

[9] Идельчик В.И. Расчеты и оптимизация режимов электрических сетей и систем. Энергоатомиздат, 1988, 287 c. [Idelchik V.I. Calculations and optimization of electrical networks and systems. Energoatomizdat, 1988, 287 p. (in Russian)]

[10] Веников В.А., Глазунов А.А., Жуков Л.А. Электрические системы. Электрические сети, М.: Высш. школа, 1998, 511 с. [Venikov V.A., Glazunov A.A., Zhukov L.A. Electrical systems. Electricity of the net, Moscow: High. school, 1998, 511 p. (in Russian)]

[11] Неуймин В.Г., Машалов Е.В., Александров А.С., Багрянцев А.А. Программный комплекс "RastrWin3”. Руководство пользователя, 2015. 240 с. [Neumin V.G., Mashalov E.V., Aleksandrov A.S., Bagryantsev A.A. Software complex “RastrWin3”. User's manual, 2015, 240 p. (in Russian)]

[12] Kim B.G., Rho D.S. Optimal voltage regulation method for distribution systems with distributed generation systems using the artificial neural networks. Journal of Electrical Engineering and Technology, 2013, 8(4), 712-718.

[13] Марикин А.Н., Мирощенко А.В., Кузьмин С.В. Устройство поперечной компенсации реактивной мощности с изменяющейся индуктивностью. Известия Петербургского университета путей сообщения, 2015, 3(44) 77-84. [Marikin A.N., Miroschenko A.V., Kuzmin S.V. Transverse reactive power compensation device with varying inductance. News of the Petersburg University of Communications, 2015, 3(44), 77-84. (in Russian)]

[14] Shahnia F., Rajakaruna S., Ghosh A. Static compensators (STATCOMs) in power systems. Springer Singapore, 2015, 175 p. 
[15] Biswas M.M., Das K.K. Voltage level improving by using static VAR compensator. Global Journal of researches in engineering J. General Engineering, 2011, 11(5) 12-18.

[16] Виноградов А. В. Голиков И.О., Бородин М.В., Бородина Е.В. Матическое регулирование напряжения на трансформаторной подстанции: способ, алгоритм и метод расчета. Промылиленная энергетика, 2014, 11, 51-55 [Vinogradov A.V. Golikov I.O., Borodin M.V., Borodina E.V. Matical voltage regulation at a transformer substation: method, algorithm and method of calculation. Industrial energy, 2014, 11, 51-55. (in Russian)]

[17] Bollen M.H.J., Hassan F. Integration of distributed generation in the power system. John wiley \& sons, 2011, 80, 510 p.

[18] Georgilakis P.S., Hatziargyriou N.D. Optimal distributed generation placement in power distribution networks: models, methods, and future research. IEEE transactions on power systems, 2013, 28(3), 3420-3428.

[19] Дмитриенко В.Н., Лукутин Б.В. Солнечно-дизельные системы электроснабжения северных поселков. Современные проблемы науки и образования, 2014, 3, 1-7 [Dmitrienko V.N., Lukutin B.V. Solar-diesel power systems of the northern villages. Modern problems of science and education, 2014, 3, 1-7. (in Russian)]

[20] Mamaghani A.H., Escandon S.A.A., Najafi B., Shirazi A., Rinaldi F. Techno-economic feasibility of photovoltaic, wind, diesel and hybrid electrification systems for off-grid rural electrification in Colombia. Renewable Energy, 2016, 97, 293-305.

[21] Саврасов Ф.В., Лукутин Б.В. Расчет эффективности использования автономных систем электроснабжения с фотоэлектростанциями на примере Томской области. Известия ТПУ, 2013, 322(6), 17-21 [Savrasov F.V., Lukutin B.V. Calculation of the effectiveness of the use of autonomous power supply systems with photopower plants on the example of the Tomsk region. News of TPU, 2013, 322(6), 17-21. (in Russian)]

[22] Стребков Д.С. Сельскохозяйственные энергетические системы и экология. Альтернативные источники энергии: эффективность и управление, 1990, 1, 39-40 [Strebkov D.S. Agricultural energy systems and ecology. Alternative energy sources: efficiency and control, 1990, 1, 39-40. (in Russian)]

[23] Mesquita F.G.G. Design optomization of stand-alone hybrid energy systems, Thesis ... cand. of tech. sci. Fevereiro de 2010, 120 p.

[24] Лукутин Б.В., Шандарова Е.Б. Энергоэффективность фотоэлектростанций в автономных системах электроснабжения. Томск: Изд-во ТПУ, 2008. 140 с. [Lukutin B.V., Shandarova E.B. Energy efficiency of photo power plants in autonomous power supply systems. Tomsk: Publishing TPU, 2008, 140 p. (in Russian)]

[25] Производитель фотоэлектрических панелей Yinglisolar [Электронный pecypc]. - Peжим доступа: http://www.yinglisolar.com/en/products/monocrystalline/ylm-60-cell-series-1/ - Заглавие с экрана. [Fabricante de painéis fotovoltaicos Yinglisolar in China [Electronic resourse] Access: http://www.yinglisolar.com/en/products/monocrystalline/ylm-60-cell-series-1/ (in Russian)] 